\title{
Qui a commandé cette pizza?
}

La situation vous est familière. Vous êtes assis au restaurant avec des amis, le sommelier a pris la commande. La conversation occupe les esprits pendant que le personnel apporte les plats. Le serveur arrive avec une pizza fumante, désigne l'assiette et cherche l'intéressé, mais personne ne réagit. Il sert les autres plats, qui trouvent preneur. A la table à côté, personne ne s'intéresse non plus au repas en rade. Il est pour le moins évident que la commande a été mal comprise ou qu'il y a eu maldonne. Que faire? Passer une nouvelle commande ou prendre la pizza préparée par erreur? On discute ferme et la bonne humeur s'en va.

Il semble en aller de même avec la banque de données sur la valeur intrinsèque. Nous présentons dans ce numéro du BMS l'état actuel de sa mise en œuvre. La commande a eu lieu en 2001 et le menu fut présenté une première fois à la «table» des assureurs à l'automne 2004. Depuis, on a vécu beaucoup de contrariétés et de stress, pesté contre qui de droit et reçu des encouragements, mais aussi vu l'équipe de cuisine, à savoir le corps médical, fournir un gros effort financier.
Depuis aussi, nous avons bien sûr contrôlé le bulletin de commande, à savoir le contrat, et pouvons assurer que le menu livré correspond à ce qu'on attendait de nous.

Il y a plusieurs possibilités de faire face à la situation:

- Laisser refroidir le repas, le jeter dans l'auge à cochons, s'énerver sur l'ignorance, l'arrogance du client, refuser la prochaine commande.

- S'asseoir avec la personne qui a passé commande les assureurs(-maladie) - et débattre de la question de savoir qui payera quelle partie des frais encourus.

Il va de soi qu'un éditorial permet de raconter (presque) n'importe quelle histoire. Celle-ci ne doit pas obligatoirement correspondre à la réalité. Si vous souhaitez connaître la véritable histoire de la banque de données sur la valeur intrinsèque, vous en trouverez un aperçu récapitulatif dans cette édition du Bulletin, à partir de la page 1528 .

Dr Olivier Kappeler, membre du Comité central de la FMH 